\title{
Baranova, J., Dingemanse, $M$. (2016). Reasons for requests. Discourse Studies, 18(6), 641-675.
}

\author{
Lilit Beganyan \\ University of California
}

Correspondence concerning this article should be addressed to Lilit Beganya, University of California, Application Center, P.O. Box 1432, Bakersfield, CA 93302, USA. E-mail: lbeganyan@rambler.ru

The article 'Reasons for Requests' written by Julija Baranova and Mark Dingemans is a profound contribution to research relating to reasoning and initiating actions, specifically providing empirical evidence for in-depth analyses. The article draws on the corpus of a six-hour and twenty-minute long video recording of everyday conversations in Russian. Most interestingly, body language is also involved in nonverbal requests. The article raises not only significant theoretical assumptions, but it has a solid theoretical framework as well.

In the Introduction of their article, the authors elucidate the target and the audience, explaining why the research focuses on the Russian language and what prior work was carried out before their study. Concepts that might be new for the audience are addressed; for example, the word 'account', which plays a fundamental role in further discussions of the topic. An account is 'a statement made by a social actor to explain unanticipated or untoward behaviour' (Scott and Lyman, 1968: 46). Moreover, the authors claim that the research will provide a deeper understanding of not only the reasons for casual interaction, but also for the usage of the Russian language as an interactional medium.

In addition, the authors define the terms and concepts that are key points of discussion. For example, 'reason' is defined as a rightful answer to the question 'why?' and 'request' is defined as an attempt to engage a recipient into a practical action. In this study, the authors specifically analyze requests concerning immediate action that is performed in the present, and the presence or absence of a suitable response to such requests. Although the authors clearly demonstrate the gap in existing literature concerning such causal interaction, they only vaguely indicate how their research plans to fill this gap.

In the 'Data and Methods' section, the authors summarize the methods used for the qualitative analysis, specifically the video recordings conducted at the participants' houses and, on two occasions, at their workplaces. In addition, around 160 verbal and nonverbal request sentences were identified, while nonverbal communication included body language with practical actions. The authors analyzed the request sentences with the help of conversational analytic methods, identifying shared sequential structures and features.

In the 'Analysis' section, the authors address: (1) requests with no reason; (2) reasons provided following a delay or problem in uptake; (3) immediate reasons that are built into the requesting turn; (4) reasons that function as a pre-request for the actual request; (5) reasons provided after compliance with the request. Following this brief overview, the paragraphs below offer a summary and analysis of each of these sections in detail.

(1) Requests with no reason imply that the request should be straightforwardly compliable and no further information is required. In their analysis, 93 out of 101 requests made were, in fact, requests without reasons. In addition to affirmations, the authors illustrate the examples through several extracts. The first starts with an offer for more tea that a host makes to her guest. The guest accepts the offer, but eventually notes that he still has some tea. Thus, Anna, the host, accepts the rejection, offering tea to another guest. When the first guest is finished his tea, he stretches his arm out and Anna pours more tea into his cup: this example clearly illustrates a request with no reason. One of the most greatest strengths of the article is that the author presents conversations in a very detailed way, displaying them not only in the original Russian, but also in its translated form, its transcription, and then analyzes each conversation line by line. Furthermore, the authors explain that under different circumstances the guest's gesture might have different options as a reply, like giving some coffee or washing the cup. They assume that this sequential embedding allows the guest to keep the conversation to a minimum while satisfying the request. The other important aspect that the authors discuss is the timing of the request. 
The example provided illustrates that the host, Anna, is still standing with the tea in her hand and so is available to pour more tea. Thus, to summarize, nonverbal requests assume that even a mere gesture or non-verbal action is enough to infer a meaning.

To make the analysis more complex and profound, the authors illustrate more complex cases involving more than two participants. However, not all requests are minimal. The second example illustrates family members gathering in kitchen. A guest offers her own son a drink and, following a positive reply, she makes a request to the host. The relation here as compared with the first example is not between requestor and requestee, but between 'requestor and benefactor of the request'. Interestingly, here with only an emphatic head nod, the recipient understands that it is a request. The goal of that conversation is to get the child a drink. As in the first case, in this one as well, the host is available for action after witnessing a conversation between a mother and a son requiring an action.

The third example is more like the first one, comprising two participants only. Lisa takes a teaspoon and her friend, Yana, is about to drink her tea and makes a request for Lisa to pass her a spoon. Lida immediately complies with the request. Thus, the authors leap to a significant conclusion that effective requests can be minimally designed and these can lead to immediate compliance. Moreover, the conversational environment fully supports the causal interaction: the proceeding talk, physical configuration of objects, and the participants' non-verbal communication make these requests fully interpretable.

The authors then move on to address requests with reasons, claiming that reasons make requests more understandable. In cases where the recipient has some trouble complying with the request, the requester can provide a reason for it to make the meaning clearer. The example provided is rather interesting in terms of empirical evidence and its complex nature. The conversation takes place in the kitchen between a mother and her two daughters. Mom is talking to one daughter who is not in the kitchen while she is putting some boiled water on the table for the other daughter. At the same time, the other daughter wants to pour instant coffee in it. Then the girls repeat a conversation over a spoon. With great accuracy, the authors mention the exact line of the confirmation, the request and the repetition. In their analysis, the reason is described as a tentative observation since the first daughter blames the mother for leaving the dirty spoon on the table. As a result the initial request was oriented toward assigning blame. The other part of their analysis involves explaining that the reason was successfully obtained by the mother's 'repair initiation'. Answering the question 'why' is very rare since, as they indicate, the question is not in harmony with common sense. The article presents several more cases of repair initiation, however, none of these directly answers the question 'why?'

An example of when the request contradicts the ongoing action of the participant is subsequently introduced. In this case, the request evokes implications which should be reinforced or rejected. These implications can be related to joking or complaining. The third conversation in this part is of utmost importance as it illustrates how the nonserious character of a request can be conveyed. The request is between school cleaners, one having dinner, the other requesting that one to serve her dinner. Through their interaction, it is clear that one of them is addressing the other in a polite form. It shows that the other woman pretends that there is a status difference between them. The next characteristic of the nonserious nature of a request is that the woman uses the imperfect imperative as opposed to its perfect version. In response here we note just a smile. The authors provide a clear description of the communication between the women by giving clues of how reason is used to make meaning clear.

At the end of this part of the article, the authors sum up their findings for this section: first, by providing a reason for the request, requesters lend specificity to requests; second, when provided, background information concerning the request can also help specify the request. In both cases, providing a reason for the request produces greater compliance.

The authors then proceed to analyze when the reason and the request are made together.

In this case the requesters provide further information concerning the request at the outset in order to avoid complications during communication. Several illustrative examples are provided, and these differ considerably from the minimal requests addressed above. They 'contradict the expectations of the previous sequence or convey delicate requests. Moreover, the requests intervene with the recipients' freedom to act upon their own wishes. The added reasons orient to this potential problem by providing grounds for such an intrusion'.

In addition, another noteworthy example occurs when a reason magnifies a complaint which is conveyed through a request. The case provided begins when friends gather for dinner. One of the guests asks the host to open the window but this request is interrupted by the other guests' conversation. We have a negative evaluation of the guest when the host fails to open the window and the guest states that carrying out the request was the least she could do; in other words, the request was simply for the host to act properly. Instead of reducing its delicacy, the reason provided over-emphasizes it. The usage of special words, such as 'the least', 'such' give extreme emphasis to the 
communication. The host complies with the request and quickly opens the window. Then one of the other guests explains why the host did not open the window immediately. In fact, 'requesters can supplement their requests with a reason that justifies this invasion and pursue compliance'. This can be followed by complaining, joking or rebuking. Thus, requests can occur together with reasons: if a speaker provides a reason for his request, he avoids misunderstanding or repetition, and the reason is immediately complied with.

The authors further analyze when a reason serves as a pre-request. In this case, Maria is sitting on the bench, obstructing the camera's view of the other woman, Katya, who addresses Maria with this issue. When Maria does not react, Katya makes a request for her to change her position. Maria's compliance is partial as she just shifts slightly on the bench for the benefit of the camera. However, as compared with the above mentioned cases the sequential relation is reversed. In this case, the request is repeated when no immediate response or compliance follows. However as the authors note, Maria cannot be held responsible for not immediately complying with the request. Only when Katya did not receive a response did she repeat her request. The analysis shows that in such cases, when reasons are used as pre-requests, the outcomes are more typical of other types of contexts.

Another interesting aspect of the communication analyzed in the article occurs when the recipient is showing compliance with the request to discover some more information. In this case a reason explains the requested action. Below is described such a case. In the communication the host asks her daughter to bring some more tea for the guests after she finished pouring the tea. The daughter complies with the mother's request, but when she goes to the kitchen a problem arises. The host's request is considered to have two parts: the first is an expectation that the daughter will bring some boiled water; the second expects that the daughter will put some water in the kettle to boil. Later it turns out that these requests have different reasons. Problems arise in communication when the daughter asks for the place of the kettle, then specifies that there is not enough water. The mother responds that not much water is needed, and then repeats her request to put the electric kettle on. We witness another problem when the daughter prepares tea which is 'too strong', and the mother repeats the expression that just a little water is enough. The mother is directly linking her request with a reason with the word because. The authors illustrate that grammatical features also contribute to the link between requests and reasons. However, explicit connectives are not that common, as reason and request are usually very close to each other. Reasons that are usually provided after the response are supposed to address by justifying or clarifying. This section is concluded by claim that after accepting reasons deal with informationally underspecified requests and ancillary actions.

In the 'Discussion and Conclusion' section, the authors sum up their findings and analysis. As they have studied reason in a sequential environment, those were very simple, face- to-face and practical forms of communication. They consider that for practical requests, the requester's ability to perform the requested action is hardly ever a problem. At the same time minimal requests could be maintained and contextualized by ongoing activities. More than one third of the requests that were used in the article had a reason in the sequence. The reasons discussed in the article were in four sequential positions, that highlighted different points at which participants dispose the need for a reason. Those four positions were: (1) reason after a delay; (2) reason with a request; (3) reason as a pre-request; (4) reason provided after acceptance. The authors conclude that despite their positional differences, the reasons had some common features; that is, reasons make the request much more understandable and expand the possibility of compliance with it. Thus, they address the under-specification of requests in three wide domains: social relations; matters of information; and action. Sometimes the context maintains the request only partially, and sometimes context contradicts the request as the latter lacks information about the required action. In such cases, reasons assist in specifying what and how the action should be done. There are also requests that interfere with the recipient's ongoing action. In these cases, the requester justifies this invasion by providing reason for it. In addition, requests can be followed by complaining, joking and rebuking. The authors concluded that despite the fact that in the literature reasons are usually associated to causal connectives like 'because', they saw such a connective in 3 cases, justifying that positioning of the reason is sufficient to relate to the request.

To conclude, reasons assist in providing understanding for requests. Reasons also deal with problematic requests in different ways: they might be informationally underspecified, delicate or potentially harmful for participants' relationships. One of the key points that the authors highlight in the article while using empirical evidence is that the causal connectives are not common, explaining it by the fact that reason and request are very close to each other. This is very significant because it shows a characteristic of the Russian language and how people communicate in everyday casual speech. The other interesting points that the authors claim and later support with empirical evidence is that when a request is allowed by a reason, 
the recipient is more likely to comply with it. This is very similar to other languages as well, as it is based on psychological and social factors. Providing a reason will solve problems and assist in the interpretation of a request. Moreover, reasons are usually a good source of information. Reasons also mitigate interpretations of a given request and emphasize other ones. In addition, reasons are multifaceted communicative mechanisms while asking for help from others.

After having reviewed in detail the background information concerning requests, the authors offer their findings and conclusions deriving from them. We come across very significant concepts in the discipline of linguistics. This article is significant in its analysis and findings as the sphere of research is rather new and there are many gaps in the current literature. Moreover, the authors carefully selected cases in the Russian language to provide empirical evidence for their research. While evaluating different aspects of communication, the authors sum up each one with their findings. The credibility of the methodological approach lies in its quantitative and qualitative analyses. However, as the nature of the analyses is highly technical, the article might be of interest to linguistics and relevant professionals dealing with it.

There is sufficient data to support the authors' generalizations. They are using specific cases for each of their empirical analyses. The cases chosen by authors are not only good examples of everyday life, but they assist if forming a firm base for further research on the topic. Future studies could be improved by continuing the research and making comparative analyses of the Russian language with some Indo-European languages, by finding the similarities and differences between two languages and trying to find the reason for these differences. The overall contribution that the article makes to the development of research and knowledge in this area is indispensable, as the topic is rather new and there is inadequate literature and research on it. 